\title{
Optimisation of asset allocation and reinsurance strategy by the mean of a genetic algorithm : NSGA-II
}

\author{
Mehdi Echchelh \\ \{mehdi.echchelh@ensae.org ${ }^{1}$ \} \\ ENSAE Paristech
}

\begin{abstract}
The management of insurance companies is a complex problem in which several indicators intervene and for which the interactions between these indicators and the management levers (reinsurance strategy, asset allocation, etc.) are not simple. We apply one of the most popular multi-objective optimization methods, namely NSGA-II. The implemented algorithm allows to find almost $98 \%$ of the hypervolume of the Pareto front in 20 iterations. In addition, the quality of representation of the Pareto front seems to be independent of the number of business of lines to be optimized, which may suggest that the work in this study may be scaled to larger companies for business use.
\end{abstract}

Keywords: Multi-objective Optimization, Genetic Algorithm, NSGA-II, Insurance, Reinsurance, Asset allocation.

\section{Introduction}

The optimization of reinsurance and asset allocation strategy is not an obvious problem as these instruments have an impact on both the profitability and the risk profile of the company. In this study, we adress the problem with one of the most popular multiobjective evolutionary algorithms, namely NSGA-II.

We note that the optimization of reinsurance treaties has already been addressed in previous work (see in particular [14], [7], [11] and [5]). The previous approaches were mainly concerned with the optimization of layers of reinsurance treaty for a given line of business. This optimization problem can not be solved analytically, thus Schlottmann, Oesterreicher and Miteschele [11] have highlighted the interest of evolutionary algorithms for this purpose.

However, to our knowledge, there is no previous work dealing with the simultaneous optimization of asset allocation and reinsurance strategy, moreover using the NSGA-II algorithm. Our work examines this problem while taking into account the usual key indicators of insurers (P\&L, ROE, Solvency Ratio, SCR).

The results obtained show that the implemented algorithm reach $98 \%$ of the hypervolume of the Pareto front in 20 iterations which is achieved in $17 \mathrm{~min}$ of computation with the hardware used in our experiments. In addition, the algorithm implemented seems to find the Pareto front independently of the number of the lines of business studied. 


\section{Reinsurance and asset allocation}

\subsection{Reinsurance}

The two types of contracts studied in this article are:

- Per risk XL (Excess of Loss): the reinsurer agrees to settle $\min (\max (S-$ $\left.\left.\min _{X L}, 0\right), \max _{X L}\right) \cdot \min _{X L}$ et $\max _{X L}$ are respectively named as the priority and the limit of the reinsurance contract. In return, the insurer must pay $P^{R}$ to the reinsurer. We set this premium as being equal to the pure premium:

$$
\mathbb{E}\left(\min \left(\max \left(S-\min _{\mathrm{XL}}, 0\right), \max _{\mathrm{XL}}\right)\right)
$$

The parameters that one seeks to optimize in the case of an XL type contract are therefore $\min _{\mathrm{XL}}$ and $\max _{\mathrm{XL}}$.

- Quota-Share: The Quota-Share reinsurance treaty is a pro rata reinsurance contract in which the reinsurer agrees to pay $\alpha_{q s} \%$ of the claims amount in return for the insurer's payment of $\alpha_{q s}\left(1-\beta_{q s}\right) \%$ of the written premiums. $\beta_{q s}$ is called the ceding commision and is subject to negotiations between insurer and reinsurer. After studying the market data, we propose the following modeling:

$$
\beta_{q s}=80 \% \max (1-S / P, 0)
$$

With $P$ and $S$ respectively the historical premium and loss amounts of the insurer. The premium perceived by the reinsurer is therefore written:

$$
P^{R}=\alpha_{q s}\left(1-\beta_{q s}\right) \cdot P
$$

The optimization of a quota-share reinsurance contract therefore lies in the optimization of the parameter $\alpha_{q s}$, also called the cession rate.

\subsection{Asset allocation}

The amount of funds to invest is divided here into three different asset classes: stocks, bonds and cash. These assets have different risk and return profile, so the choice of the amounts invested in each of these assets will impact both the risk and the profitability of the whole portfolio. Generally French insurers invest from $80 \%$ to $90 \%$ of their assets in bonds (see [1], [2], and [3]), which is considered a low-risk investment but produces a lower return than equities. 


\section{Methods}

\subsection{Objectives mapping}

We define in the following two parts the objective function of our optimization problem, namely:

$$
F: \omega \in \Omega \rightarrow\left(f_{1}(\omega), \ldots f_{n}(\omega)\right)
$$

With $\Omega$ the decision space.

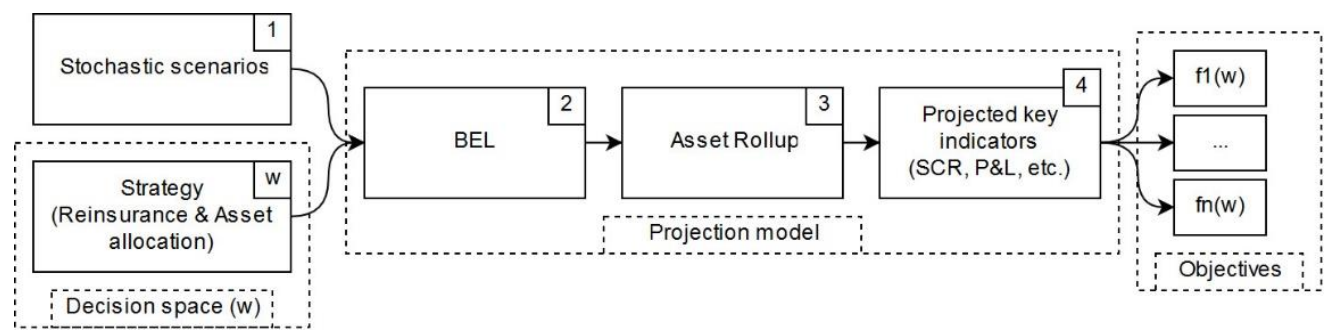

Fig. 1. Objectives mapping.

The calculation of objective values relies on stochastic simulations and on a projection model giving the distribution of different key indicators (P\&L, ROE, etc.). Projection models are commonly used in insurance, they enable the assessment of business plan impact on the projection of key indicators. A projection model is defined over a fixed time horizon and with a specific time step, in this study we use a 5 years projection with an annual time step.

The projection model implemented here can be broken down into four stages wich are represented on the figure Figure 1. [1]:

1. Simulation of stochastic scenarios: we simulate claims' amounts for each line of business as well as the main economic variables (zero-coupon rate, stock return). These scenarios are then used to compute the projection of liabilities and assets (see steps 2 and 3 below). In this study we simulate 1000 stochastic scenarios.

2. Projection of liabilities: under the Solvency II regulatory framework the insurer's liabilities are composed of three elements: the Best Estimate Liabilities (BEL) which is the present value of the insurer's undertaking, the Risk Margin (RM) which we take proportional to the BEL in order to reduce the calculation time and the Net Asset Value, which is calculated as the difference between the market value of the assets and the sum of the BEL and the RM.

3. Projection of assets: once the first two steps have been completed, we project the market value of the company's investments. This projection is carried out by iteratively projecting the market value of the investments, taking into account the evolution of the economic variables, the claims payments as well as the allocation strategy of the company. 
4. Calculation of the key indicators: Once steps 1,2 and 3 have been completed, we calculate the value of the SCR and the different key indicators for each projection year and simulation.

\subsection{Objectives and constraints}

The defined projection model allows us to obtain the distributions of specific key indicators. The objectives are then defined by applying certain statistics (mean, standard deviation, VaR or quantile, etc.) on these indicators.

In the following we will consider two objectives, namely:

- $\mathbb{E}(P \& L)$

- $\mathbb{E}(S C R)$

The constraints taken into account - so called risk appetite constraints - are defined by:

- $\quad \operatorname{VaR}_{10 \%}^{5 \text { years }}(R O E)>1 \%$

- $\quad \operatorname{VaR}_{10 \%}^{5}$ years $($ Solvency Ratio) $>200 \%$

- $\operatorname{VaR}_{90 \%}^{5 \text { years }}(S / P)<100 \%$

Let us note that the expectation and the $\mathrm{VaR}$ are estimated by using the empirical average and the empirical quantile on the outputs from the projection model.

\subsection{Optimisation algorithm}

Evolutionary algorithms are naturally suitable algorithms for the optimisation problem studied here since they do not require any assumptions of derivability or convexity of the objective functions (see [9]). Hence we use one of the most popular algorithm, namely NSGAII.

NSGA-II provides a so-called "truly" multi-objective approach, as it makes it possible to simultaneously optimize several objectives and thus to find the entire Pareto front. As for all genetic algorithms, NSGA-II relies on genetic operators. The crossover and mutation operators used in this study are respectively SBX (Simulated binary Crossover) and polynomial mutation. The interested reader may refer to Kalyanmoy Deb et al. for more details (see [12]).

Appication to asset allocation and reinsurance optimisation. The crossover and mutation operators initially proposed by K. Deb for the NSGA-II algorithm are defined for real-coded individuals represented by vectors of $[0,1]^{n}$, with $n$ the number of parameters or "genes". This representation suits perfectly for the optimization of the quantitative parameters (asset allocation, priority and limit of XL treaties). However when taking into account qualitative parameters such as the choice between an XL or a Quota-Share coverage, the real-coded representation of individuals doesn't naturally fit. The solution implemented here is to enumerate all the possible choice for each qualitative parameter and then to optimize the quantitative parameters for each of these choices. The algorithm is as follows:

- Enumerate all possible choices for the types of reinsurance coverage. If we have $N_{l o b}$ lines of business and $n$ different types of reinsurance contracts, the number of possibilities is $N_{l o b}^{n}$. 
- For each choice previously enumerated, we optimize the quantitative parameters with the algorithm NSGA-II launched on a parallel worker.

The calculation in this study were done on a computer equiped with an intel i7 $8^{\text {th }}$ generation processor with 6 cores. So by using the package future of R, we parallelized calculations on the 6 cores, which improved the computation time by a factor of 4 .

Dealing with constraints. To take into account the constraints of the optimization problem, we have chosen one of the most popular approach, namely the "multi-objective transformation" approach. It consists in integrating the constraints into the objectives set, thus it preserves the multi-objective aspect of the problem.

The optimization problem is thus as follows:

$$
\begin{aligned}
\min & +\mathbb{E}(P \& L), \\
& -\mathbb{E}(S C R), \\
& +\operatorname{VaR}_{10 \%}^{5 \text { years }}(\text { ROE }), \\
& +\operatorname{VaR}_{10 \%}^{5 \text { years }}(\text { Solvency Ratio }), \\
& \left.-\operatorname{VaR}_{90 \%}^{5 \text { years }}(S / P)\right)
\end{aligned}
$$

\section{Application and results}

\subsection{Impact of hyperparameters on normalized hypervolume}

In this section we study the impact of the number of generations and of the population size on the quality of the Pareto front approximation, which is measured by the normalised relative hypervolume (NRHV).

Number of generations. The Figure 2. [2] gives the evolution of the average NRHV over 30 runs of the optimization algorithm. We observe after the first 20 iterations that the difference between the $N R H V$ of the population and that of the reference front is less than $2.3 \%$. We notice that the iteration 21 is reached on average after $17 \min 24 \mathrm{~s}$, which can be considered as a reasonable time for a business use.

NB. - The reference frontier corresponds to the set of non-dominated stratgies obtained on all the runs. The number of non-dominated strategies is 3,777. 


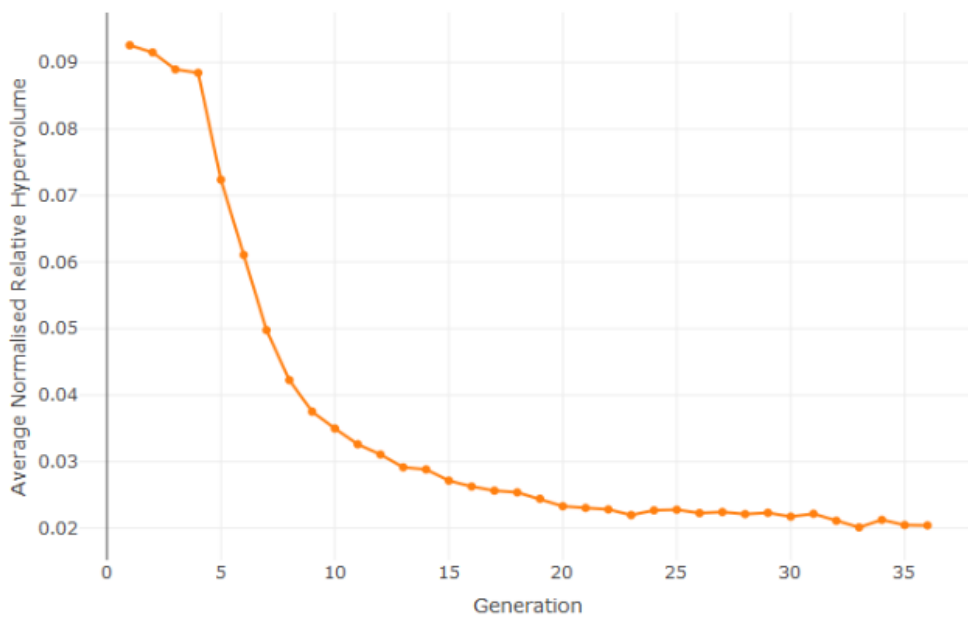

Fig. 2. Evolution of normalized relative hypervolume with the number of iterations.

Population size. We observe in the figure 3 that the normalized relative hypervolume does not seem to be able to decree until 0 . This is because the number of individuals is a limit to the quality of the Pareto front approximation. The Figure 3. [3] gives an illustration of the impact of the population size on the quality of representation of the Pareto front.

The increase in population size shifts the whole curve downwards. However, in the proposed implementation, the calculation time increases linearly with the number of individuals. Thus the choice of the population size should result in practice from an trade-off between calculation cost and quality of representation of the Pareto front.

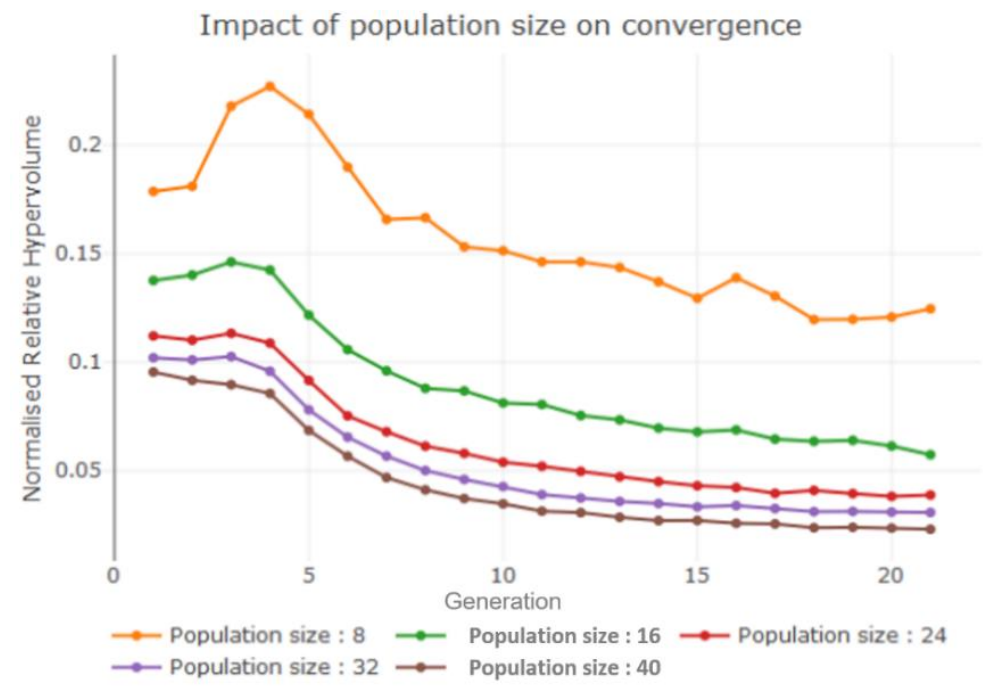

Fig. 3. Impact of population size on convergence. 


\subsection{Impact of the number of LOB on normalized hypervolume}

We study the impact of the number of lines of business on the Pareto front approximation quality. So we optimize the company's strategy in the case where it reinsures one, two or three different business lines. The Figure 4. [4] does not show obvious differences between the three curves, besides a one-way ANOVA test allows us to conclude that one can not reject the hypothesis of equality of the Pareto front approximation quality for one, two or three lines of business ( $p$-value : $0.27>0.05$ ).

So the quality of the the Pareto front approximation seems independent of the number of lines of business considered in the problem.

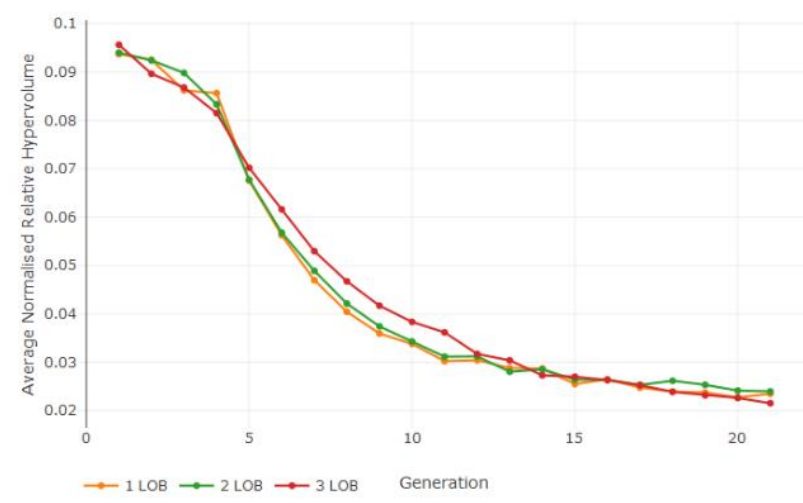

Fig. 4. Impact of the number of business lines on convergence.

\subsection{Analysis of the relevance of the Pareto Front}

In this section we analyse the Pareto front obtained with the optimization algorithm. The company considered consists of three lines of business and has three assets to its portfolio and its optimization problem is given by the equation (4).

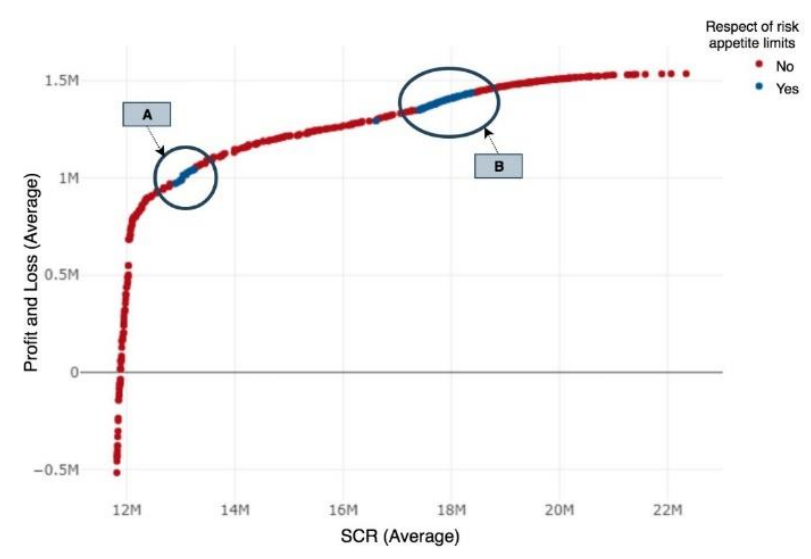

Fig. 5. Illustration of the Pareto front obtained. 
On Figure 5. [5] we represent the Pareto front. The points for which the constraints are respected are shown in blue, while the points for which the constraints are not respected are shown in red. We observe that the Pareto front has two zones for which the risk appetite constraints are respected, the table 1 gives an overview of these strategies. By comparing the asset allocation strategies obtained with the algorithm with the allocations observed on the French insurance market, we see that the amounts of equity, bonds and cash are in similar proportions for type B strategies, which reinforces the relevance of the projection model and the results obtained with the optimization algorithm.

Table 1. Overview of optimal strategies. Strategies 1 and 2 belong to set A, strategy 3 and 4 belong to set $\mathrm{B}$.

\begin{tabular}{|l|ll|lll|lll|}
\hline \multirow{2}{*}{$\mathrm{N}$} & \multirow{2}{*}{$\mathbb{E}(P \& L)$} & $\mathbb{E}(S C R)$ & \multicolumn{3}{|l|}{ Asset Allocation } & \multicolumn{3}{l|}{ Reinsurance } \\
\cline { 4 - 8 } & & Equity & Bond & Cash & LOB 1 & LOB 2 & LOB 3 \\
\hline 1 & $976 \mathrm{k}$ & $12.9 \mathrm{M}$ & $1 \%$ & $95 \%$ & $0 \%$ & $460 \mathrm{k}$ XL 5k & QS: 75\% & QS: 17\% \\
2 & $1.01 \mathrm{M}$ & $13 \mathrm{M}$ & $3 \%$ & $96 \%$ & $1 \%$ & $840 \mathrm{k}$ XL 5k & QS: 73\% & QS: 15\% \\
\hline 3 & $1.35 \mathrm{M}$ & $17 \mathrm{M}$ & $8 \%$ & $90 \%$ & $1 \%$ & $711 \mathrm{k}$ XL 5k & $328 \mathrm{k}$ XL 9k & 430k XL 71k \\
4 & $1.43 \mathrm{M}$ & $18.3 \mathrm{M}$ & $16 \%$ & $83 \%$ & $1 \%$ & 941k XL 5k & $856 \mathrm{k}$ XL 9k & 466k XL 57k \\
\hline
\end{tabular}

Table 2. Asset allocation of French insurers. Shares invested in equities, bonds and cash by French insurers. Source : ACPR (see [ ]).

NB. $-M_{i}$ refers to the amount invested in asset $i$.

\begin{tabular}{|c|c|c|c|}
\hline Semester & $\frac{M_{\text {stock }}}{M}$ & $\frac{M_{\text {bond }}}{M}$ & $\frac{M_{\text {cash }}}{M}$ \\
\hline December -2017 & $13 \%$ & $84 \%$ & $3 \%$ \\
\hline December - 2016 & $13 \%$ & $82 \%$ & $5 \%$ \\
\hline
\end{tabular}

\section{Conclusion}

Finally, the proposed method highlighted the benefit of evolutionary algorithms in insurance and more particularly in the decision-making process. This type of study is all the more important now that the regulatory context is becoming more and more restrictive for the management of insurance companies.

The implemented algorithm reached $98 \%$ of the hypervolume of the reference front after 20 iterations, which was achieved in $17 \mathrm{~min}$ with a consumer laptop, equipped with an Intel processor i7 8700k. The method implemented here seems therefore relevant in business-use applications, however we note that the proposed parallelization reach its limits when the number of lines of busines becomes too large in comparison to the number of cores of the processor.

In addition, we observed that the quality of representation of the Pareto front is the same when we consider 1, 2 or 3 lines of business. It therefore seems that the proposed approach can be generalized and still offer a good representation of the Pareto front when the number of business lines becomes larger. 
However it is important to note that the projection model developed is based on certain assumptions and may require adjustments in a real application. In addition, we have restricted ourselves to the optimization of two objectives - apart from risk appetite constraints - in order to facilitate the visualization of the Pareto front. It might be interesting to explore multicriteria analysis methods to guide decisions when the Pareto front can not be viewed in its entirety. 


\section{References}

[1] ACPR: La situation des assureurs soumis à Solvabilité II en France au premier semestre 2017. (2017)

[2] ACPR: La situation des assureurs soumis à Solvabilité II en France au premier semestre 2018. (2018)

[3] ACPR: La situation des assureurs soumis à Solvabilité II en France à fin 2017. (2018)

[4] Omar Carmona Cortes and Andrew Rau-Chaplin.: Enhanced multiobjective population-based incremental learning with applications in risk treaty optimization. Evolutionnary Intelligence (2016)

[5] Omar Carmona Cortes and Andrew Rau-Chaplin.: Reinsurance analytics using serial and parallel computation on the multiobjective evolutionary algorithm SPEA2. (2018)

[6] Omar Carmona Cortes and Andrew Rau-Chaplin.: Tackling Reinsurance Contract Optimization by Means of Evolutionary Algorithms and HPC. (2018)

[7] Omar Carmona Cortes and Andrew Rau-Chaplin, Pedro Felipe Do Prado.: A new vector evaluated PBIL algorithm for reinsurance analytics. (2015)

[8] Omar Carmona Cortes and Andrew Rau-Chaplin, Rafael Ferandes Lopes.: A survey on reinsurrance contract optimization using evolutionary and swarm computation. IEEE Latin America Transactions. (2016)

[9] Van Veldhuizen Coello, Lamont.: The book of MOEA: Evolutionary Algorithms for Solving MultiObjective Problems. (2015)

[10] Detlef Seese Frank Schlottmann, Andreas Mitschele.: A multi-objective approach to integrated risk management.

[11] F. Schlottmann. I. Oesterreicher, A. Mitschele.: Comparison of multiobjective evolutionary algorithms in optimizing combinations of reinsurance contracts.

[12] Sameer Agarwal-T. Meyarivan Kalyanmoy Deb, Amrit Pratap.: fast and elitist multiobjective genetic algorithm: NSGA-II. (2002)

[13] Journal officiel de l'Union européenne. Règlement délégué (UE) 2015/ 35 de la commission - du 10 octobre 2014.

[14] Duane Wilson, Jürgen Gaiser-Porter, Omar Andres Carmona Cortes, Andrew Rau-Chaplin.: On PBIL, DE and PSO for optimization of reinsurance contracts. (2014) 